Mathematical Research Letters 2, 823-829 (1995)

\title{
$B$-STRUCTURES ON $G$-BUNDLES AND LOCAL TRIVIALITY
}

\section{G. Drinfeld and Carlos Simpson}

1. Let $G$ be a split reductive group scheme over $\mathbb{Z}$ (recall that for any algebraically closed field $k$ there is a bijection $G \mapsto G \otimes k$ between isomorphism classes of such group schemes and isomorphism classes of connected reductive algebraic groups over $k$ ). Let $B$ be a Borel subgroup of $G$. Let $S$ be a scheme and $X$ a smooth proper scheme over $S$ with connected geometric fibers of pure dimension 1 . Our goal is to prove the following theorems.

Theorem 1. Any $G$-bundle on $X$ admits a B-structure after a suitable surjective etale base change $S^{\prime} \rightarrow S$.

Theorem 2. Any G-bundle on $X$ becomes Zariski-locally trivial after a suitable etale base change $S^{\prime} \rightarrow S$.

Theorem 3. Suppose that $G$ is semisimple. Let $D$ be a subscheme of $X$ such that the projection $D \rightarrow S$ is an isomorphism. Set $U:=X \backslash D$. Then for any $G$-bundle $F$ on $X$ its restriction to $U$ becomes trivial after a suitable faithfully flat base change $S^{\prime} \rightarrow S$ with $S^{\prime}$ being locally of finite presentation over $S$. If $S$ is a scheme over $\mathbb{Z}\left[n^{-1}\right]$ where $n$ is the order of $\pi_{1}(G(\mathbb{C}))$ then $S^{\prime}$ can be chosen to be etale over $S$.

2. Remarks. a) Theorem 2 follows from Theorem 1 because a $B$ bundle on any scheme is Zariski-locally trivial.

b) If $S$ is the spectrum of an algebraically closed field Theorems 1-3 are well known (of course in this case base change is not necessary). In this situation Theorem 3 was proved in [6], while Theorems 1 and 2 follow from the triviality of $G$-bundles over the generic point of $X$. The triviality of the Galois cohomology $H^{1}(k(X), G)$ was conjectured by J. P. Serre and proved by R. Steinberg [9] and A. Borel and T. A. Springer [3]. Note that Steinberg's result is for a perfect field of dimension 1-inconvenient here since $k(X)$ is not perfect in characteristic $p$ (whereas of course it is of dimension 1). The restriction to perfect fields was due to the need to have $G$

Received October 9, 1995.

The first author was partially supported by INTAS grant No.94-4720 and DKNT grant No. 11.3/21. 
split, which doesn't matter here, and the need to apply Rosenlicht's density theorem. The density theorem was improved by A. Grothendieck [4] and A. Borel and T.A. Springer ([2] Theorem A) to eliminate the perfect hypothesis. The resulting stronger version of Steinberg's result is announced in [3] section 8.6.

c) Theorem 3 is an answer to a question by A. Beauville and Y. Laszlo. They proved Theorem 3 for $G=\mathrm{SL}(n)$ ([1], Lemma 3.5). They showed that in this case it is enough to localize with respect to the Zariski topology of $S$. Theorem 3 is used in $[1,7]$ to prove the "uniformization" theorem.

d) Since we localize with respect to the etale topology of $S$ the splitness assumption on $G$ is not essential in Theorems 1-3 (in the situation of Theorem 1 one can define the notion of $B$-structure even if $G$ has no Borel subgroups).

e) If $S$ is a scheme over an algebraically closed field $k$ then Theorems 1-3 hold for $\mathcal{G}$-bundles where $\mathcal{G}$ is a connected affine algebraic group over $k$ which in the case of Theorem 3 must have the property $\operatorname{Hom}\left(\mathcal{G}, G_{m}\right)=0$. This easily follows from Theorems 1-3 for $G$-bundles (the unipotent radical of $\mathcal{G}$ does not matter).

3. Theorem 1 for $G$ follows from Theorem 1 for $G / Z^{0}$ where $Z^{0}$ is the connected component of the center $Z \subset G$. The same holds for Theorem 2 , which also follows from Theorem 1 . So we will suppose hereinafter that $G$ is semisimple. We will also assume that all the fibers of $X \rightarrow S$ have the same genus $g$.

4. Fix a Cartan subgroup $H \subset B$. Let $\alpha_{i}: H \rightarrow G_{m}, i \in \Delta$, be the simple roots. Denote by $\bar{\alpha}_{i}$ the corresponding morphisms $B \rightarrow G_{m}$. For a $B$-bundle $E$ on a smooth projective curve over a field denote by $\operatorname{deg}_{i}(E)$ the degree of the $G_{m}$-bundle associated to $E$ and $\bar{\alpha}_{i}$.

Let $F$ be a principal $G$-bundle on $X$ ( $G$ acts on the scheme $F$ from the right). $B$-structures on $F$ can be identified with sections of $F / B \rightarrow X$. Consider the functor $\Phi$ that associates to a scheme $T$ over $S$ the set of $B$-structures on $F \times_{S} T$ considered as a $G$-bundle on $X \times_{S} T$. Identifying a section with its graph, the theory of Hilbert schemes shows that $\Phi$ is representable by a scheme $M_{F}$ locally of finite presentation over $S$. To a point $y \in M_{F}$ there corresponds a $B$-bundle $E_{y}$ on the fiber of $X$ over the image of $y$ in $S$. Set $d_{i}(y):=\operatorname{deg}_{i}\left(E_{y}\right)$. The functions $d_{i}$ are locally constant. Set $M_{F}^{+}:=\left\{y \in M_{F}: d_{i}(y)<\min (1,2-2 g), i \in \Delta\right\}$.

Proposition 1. The morphism $M_{F}^{+} \rightarrow S$ is smooth.

Proposition 2. The morphism $M_{F}^{+} \rightarrow S$ is surjective. 
These propositions imply that for some surjective etale morphism $S^{\prime} \rightarrow$ $S$ there exists an $S$-morphism $S^{\prime} \rightarrow M_{F}$. This is precisely Theorem 1 .

Proof of Proposition 1. Let $k$ be a field, $y \in M_{F}(k)$. Let $s \in S(k)$ be the image of $y$ and $X_{s}$ the fiber of $X$ over $s$. The point $y$ corresponds to a section $\sigma: X_{s} \rightarrow F / B$ of the morphism $F / B \rightarrow X$. Standard deformation theory shows that the morphism $M_{F} \rightarrow S$ is smooth in a neighbourhood of $y$ provided $H^{1}\left(X_{s}, \sigma^{*} \Theta\right)=0$ where $\Theta$ denotes the relative tangent bundle of $F / B$ over $X . \sigma^{*} \Theta$ is the vector bundle associated to the $B$-bundle $E_{y}$ on $X_{s}$ corresponding to $y$ and the $B$-module $\operatorname{Lie}(G) / \operatorname{Lie}(B)$. So it is easy to see that if $\operatorname{deg}_{i}\left(E_{y}\right)<\min (1,2-2 g)$ for all $i \in \Delta$ then $H^{1}\left(X_{s}, \sigma^{*} \Theta\right)=0$, Q.E.D.

5. The following statement is stronger than Proposition 2.

Proposition 3. Let $Y$ be a smooth projective curve over an algebraically closed field $k$ and $F$ a G-bundle on $Y$. Then for any number $N$ there is a B-structure on $F$ such that for the corresponding B-bundle $E$ one has $\operatorname{deg}_{i}(E)<-N$ for all $i \in \Delta$.

Proof. 1) It does not matter which $F$ to consider. Indeed, according to [9] [3] a $G$-bundle on $Y$ is trivial over the generic point of $Y$. So if $F$ and $F^{\prime}$ are $G$-bundles on $Y$ there is an isomorphism $h$ between the restrictions of $F$ and $F^{\prime}$ to $Y \backslash R$ for some finite $R \subset Y$. If $h$ is fixed then a $B$-structure on $F$ induces a $B$-structure on $F^{\prime}$ and for the corresponding $B$-bundles $E$ and $E^{\prime}$ the inequalities $-c<\operatorname{deg}_{i}(E)-\operatorname{deg}_{i}\left(E^{\prime}\right)<c$ hold for some $c$ depending only on the singularities of $h$.

2) So we can assume that $F$ is trivial.

3) Since $F$ is trivial we can assume that $G$ is simply connected (otherwise the inverse image $B^{\prime}$ of $B$ in the universal covering $G^{\prime}$ is a Borel; let $F^{\prime}$ be the trivial $G^{\prime}$-bundle, then a $B^{\prime}$-bundle which induces the $G^{\prime}$-bundle $F^{\prime}$ must induce a $B$-bundle which induces $F$ ) and $Y=P^{1}$ (choose a finite morphism $f: Y \rightarrow P^{1}$ and consider those $B$-structures on the trivial $G$-bundle on $Y$ which are pullbacks via $f$ of $B$-structures on the trivial $G$-bundle on $P^{1}$ ).

4) Denote by $\operatorname{Bun}_{B}$ the stack of $B$-bundles on $P^{1}$. Let $\operatorname{Bun}_{B}^{n}$ be the open subset of $\operatorname{Bun}_{B}$ corresponding to $B$-bundles $E$ such that $\operatorname{deg}_{i}(E)=n$ for all $i \in \Delta$. There is an integer $m>0$ such that $\operatorname{Bun}_{B}^{n} \neq \emptyset$ if $m \mid n$. Choose $n$ so that $\operatorname{Bun}_{B}^{n} \neq \emptyset, n<-N, n<0$. According to Proposition 1 the natural morphism $\operatorname{Bun}_{B}^{n} \rightarrow \operatorname{Bun}_{G}$ is smooth. So its image $U$ is open and non-empty. Since $Y=P^{1}$ trivial $G$-bundles form an open substack $V$ of $\operatorname{Bun}_{G}$. Since $G$ is simply connected $\mathrm{Bun}_{G}$ is irreducible (see Appendix). So $U \cap V \neq \emptyset$, Q.E.D. 
Remark. If char $k=0$ one can replace steps 3 and 4 of the above proof by the following argument. There is a homomorphism $r: \operatorname{SL}(2) \rightarrow G \otimes Q$ such that 1) $r$ maps diagonal matrices to $H \otimes Q$ and upper-triangular matrices to $B \otimes Q, 2) \alpha_{i}\left(r\left(\operatorname{diag}\left(t, t^{-1}\right)\right)=t^{m_{i}}, i \in \Delta\right.$, where $m_{i}$ are positive (e.g., the "principal" homomorphism SL $(2) \rightarrow G \otimes Q$ has these properties). Using $r$ one reduces the problem for the trivial $G$-bundle to that for the trivial SL(2)-bundle. The latter problem is trivial: if $G=\mathrm{SL}(2)$ then a $B$ structure on the trivial $G$-bundle is just a morphism $f: Y \rightarrow(G / B) \otimes k=$ $P_{k}^{1}$ and the only condition is that $\operatorname{deg} f$ should be big enough. We do not know whether this argument works if char $k \neq 0$.

6. Proof of Theorem 3. We can assume that $S$ is affine. According to Theorem 1 one can also suppose that $F$ comes from a $B$-bundle $E^{\prime}$. Denote by $E$ the $H$-bundle obtained from $E^{\prime}$ via the homomorphism $B \rightarrow H$ and by $F_{1}$ the $G$-bundle coming from $E$. Since $U$ is affine $E^{\prime}$ and the $B$-bundle obtained from $E$ via the embedding $H \rightarrow B$ are isomorphic over $U$. So the restrictions of $F$ and $F_{1}$ to $U$ are isomorphic. Therefore one can assume that $F$ comes from an $H$-bundle $E$.

Let us reduce the problem to the case where $G$ is simply connected. Set $A=\operatorname{Hom}\left(H, G_{m}\right)$. $E$ defines a section $u: S \rightarrow \operatorname{Hom}(A$, Pic $X)$ where Pic $X$ denotes the relative Picard scheme of $X / S$ and Hom denotes the scheme of homomorphisms. One can assume that $u(S) \subset \operatorname{Hom}\left(A, \operatorname{Pic}^{0} X\right)$ (otherwise change $E$ without changing its restriction to $U$ ). Let $G^{\prime}$ be the universal cover of $G, H^{\prime}$ the preimage of $H$ in $G^{\prime}, A^{\prime}:=\operatorname{Hom}\left(H^{\prime}, G_{m}\right)$. A is a subgroup of $A^{\prime}$ and its index is equal to the order $n$ of $\operatorname{Ker}\left(G^{\prime} \rightarrow G\right)$. The morphism $\operatorname{Hom}\left(A^{\prime}, \operatorname{Pic}^{0} X\right) \rightarrow \operatorname{Hom}\left(A, \operatorname{Pic}^{0} X\right)$ is flat, finite, and of finite presentation; if $S$ is a scheme over $\mathbb{Z}\left[n^{-1}\right]$ it is etale. Denote by $S^{\prime}$ the fibered product of $S$ and $\operatorname{Hom}\left(A^{\prime}, \operatorname{Pic}^{0} X\right)$ over $\operatorname{Hom}\left(A, \operatorname{Pic}^{0} X\right)$. The pullback of $E$ to $X \times_{S} S^{\prime}$ can be lifted to an $H^{\prime}$-bundle locally with respect to the Zariski topology of $S^{\prime}$.

So one can assume that $G$ is simply connected and $F$ comes from an $H$-bundle $E$. Since $G$ is simply connected $\operatorname{Hom}\left(G_{m}, H\right)$ is freely generated by simple coroots. So it suffices to show that if $H$ - bundles $E_{1}$ and $E_{2}$ differ by the image of some $G_{m}$-bundle via a coroot $\check{\alpha}: G_{m} \rightarrow H$ then the $G$-bundles corresponding to $E_{1}$ and $E_{2}$ are isomorphic locally over $S$. In fact we will show that it is true for $G$ replaced by the subgroup $L \subset G$ generated by $H$ and $r(\mathrm{SL}(2))$ where $r: \mathrm{SL}(2) \rightarrow G$ corresponds to $\check{\alpha}$. It is easy to show that either $L=\mathrm{SL}(2) \times T$ or $L=\mathrm{GL}(2) \times T^{\prime}$ where $T$ and $T^{\prime}$ are tori. In the first case it suffices to show that the restriction to $U$ of an $\mathrm{SL}(2)$-bundle on $X$ is trivial locally over $S$. In the second case it is enough to show that that the restriction to $U$ of two GL(2)-bundles on $X$ with the same determinant are isomorphic locally over $S$. The first 
statement is proved by Beauville-Laszlo ([1] , Lemma 3.5). The proof of the second statement is quite similar (in both cases Zariski localization is enough).

7. In the proof of Theorems 2 and 3 we used Theorem 1. Actually we could use the following weaker version of Theorem 1, which can be proved without using Propositions 2 and 3.

Proposition 4. In the situation of Theorem 3 for any $G$-bundle $F$ on $X$ there is a surjective etale base change $S^{\prime} \rightarrow S$ and a $G$-bundle $F^{\prime}$ on $X \times{ }_{S} S^{\prime}$ such that the inverse images of $F$ and $F^{\prime}$ on $U \times{ }_{S} S^{\prime}$ are isomorphic and $F^{\prime}$ has a $B$-structure.

Proof (inspired by [5] p. 364, lines 26-34). We can assume that $S$ is strictly henselian, i.e., $S$ is the spectrum of a strictly henselian ring (in this case base change is not necessary). Let $s$ be the closed point of $S$, $X_{s}$ the fiber of $X$ over $s$, and $F_{s}$ the restriction of $F$ to $X_{s}$. According to [9] [3] the restriction of $F_{s}$ to the generic point of $X_{s}$ is trivial. So $F_{s}$ has a $B$-structure, i.e., it comes from a $B$-bundle $E$ on $X_{s}$. Since the restriction of $E$ to $U_{s}:=U \cap X_{s}$ comes from an $H$-bundle it is easy to construct a $B$-bundle $E^{\prime}$ on $X_{s}$ such that the restrictions of $E$ and $E^{\prime}$ to $U_{s}$ are isomorphic and $\operatorname{deg}_{i}\left(E^{\prime}\right)<\min (1,2-2 g$ ) for all $i \in \Delta$ (see Section 4 for the definition of $\operatorname{deg}_{i}$ ). Denote by $F_{s}^{\prime}$ the $G$-bundle on $X_{s}$ corresponding to $E^{\prime}$. The restrictions of $F_{s}^{\prime}$ and $F_{s}$ to $U_{s}$ are isomorphic. We will construct a $G$-bundle $F^{\prime}$ on $X$ such that the restrictions of $F$ and $F^{\prime}$ to $U$ are isomorphic and the restriction of $F^{\prime}$ to $X_{s}$ is equal to $F_{s}$. According to Proposition 1 such $F^{\prime}$ automatically has a $B$-structure.

For every scheme $\tilde{X}$ etale over $X$ denote by $M(\tilde{X})$ the set of isomorphism classes of pairs consisting of a $G$-bundle $F^{\prime}$ on $\tilde{X}$ and an isomorphism $f$ between the inverse images of $F$ and $F^{\prime}$ on $U \times_{X} \tilde{X}$. Then $M(\tilde{X})=H^{0}\left(\tilde{X}, j_{*} j^{*} \mathcal{G} / \mathcal{G}\right)$ where $\mathcal{G}$ is the (etale) sheaf of automorphisms of $F$ and $j: U \hookrightarrow X$. (To see this note first that pairs $\left(F^{\prime}, f\right)$ have no nontrivial automorphisms, consequently that $M$ is the etale sheaf associated to the presheaf $M_{0}$ where $M_{0}(\tilde{X}) \subset M(\tilde{X})$ is the subset of pairs $\left(F^{\prime}, f\right)$ with $F^{\prime}$ isomorphic to the inverse image of $F$, and finally that $M_{0}(\tilde{X})=H^{0}\left(\tilde{X}, j_{*} j^{*} \mathcal{G}\right) / H^{0}(\tilde{X}, \mathcal{G})$.) In particular we have $M(X)=$ $H^{0}\left(X, j_{*} j^{*} \mathcal{G} / \mathcal{G}\right)=H^{0}\left(D, i^{*}\left(j_{*} j^{*} \mathcal{G} / \mathcal{G}\right)\right)$ where $i: D \hookrightarrow X$. Since $D \simeq S$ is strictly henselian $M(X)=\left(j_{*} j^{*} \mathcal{G}\right)_{w} / \mathcal{G}_{w}$ where $w$ is the point of $D$ over $s$ and $\mathcal{G}_{w}$ is the stalk of $\mathcal{G}$ at $w$. Fix a trivialization of $F$ over an etale neighbourhood of $w$ and a local equation $t=0$ defining $D \subset X$. These data define isomorphisms $\mathcal{G}_{w}=G(A\{t\}),\left(j_{*} j^{*} \mathcal{G}\right)_{w}=G\left(A\{t\}\left[t^{-1}\right]\right)$ where $A=H^{0}\left(S, O_{S}\right)$ and $A\{t\}$ is the henselization of $A[t]$ at the maximal ideal containing $t$. $F_{s}$ defines an element of $G\left(k\{t\}\left[t^{-1}\right]\right) / G(k\{t\})$ 
where $k$ is the residue field of $A$, and we have to lift it to an element of $G\left(A\{t\}\left[t^{-1}\right]\right) / G(A\{t\})$. This can be done, e.g., using the Iwasawa decomposition $G\left(k\{t\}\left[t^{-1}\right]\right)=B\left(k\{t\}\left[t^{-1}\right]\right) G(k\{t\})$.

\section{Appendix}

Let $Y$ be a smooth connected projective curve over an algebraically closed field $k$. Denote by $\operatorname{Bun}_{G}$ the stack of $G$-bundles on $Y$. The following statement is well known but we could not find a reference with a proof valid for all $G$ without the assumption char $k=0$.

Proposition 5. If $G$ is simply connected then $\operatorname{Bun}_{G}$ is irreducible.

Proof. A well known deformation-theoretic argument shows that $\mathrm{Bun}_{G}$ is smooth, so it suffices to show that $\mathrm{Bun}_{G}$ is connected. The morphism $\operatorname{Bun}_{B} \rightarrow \operatorname{Bun}_{G}$ is surjective, so the mapping $\pi_{0}\left(\operatorname{Bun}_{B}\right) \rightarrow \pi_{0}\left(\operatorname{Bun}_{G}\right)$ is also surjective. The mapping $\pi_{0}\left(\mathrm{Bun}_{H}\right) \rightarrow \pi_{0}\left(\mathrm{Bun}_{B}\right)$ is bijective (use the homomorphism $B \rightarrow H$ to prove injectivity; surjectivity follows from the existence of a family of endomorphisms $f_{t}: B \rightarrow B, t \in A^{1}$, such that $f_{1}=$ id and $f_{0}(B)=H$ : just set $f_{t}(b):=\lambda(t) b \lambda(t)^{-1}$ where $\lambda \epsilon$ $\operatorname{Hom}\left(G_{m}, H\right)$ is a strictly dominant coweight). Since $G$ is simply connected $\pi_{0}\left(\operatorname{Bun}_{H}\right)=\operatorname{Hom}\left(G_{m}, H\right)$ can be identified with the coroot lattice. So a standard argument (see, e.g., the end of Section 6) shows that it suffices to prove the connectedness of the stack of rank 2 vector bundles with a fixed determinant $\mathcal{M}$. Given two such vector bundles $L_{1}$ and $L_{2}$ there is a line bundle $\mathcal{A}$ such that $L_{1} \otimes \mathcal{A}$ and $L_{2} \otimes \mathcal{A}$ are generated by their global sections. Then according to a lemma due to Serre ([8], p.27, Lemma 24) there exist exact sequences

$$
0 \rightarrow \mathcal{A}^{\otimes(-1)} \rightarrow L_{i} \rightarrow \mathcal{M} \otimes \mathcal{A} \rightarrow 0, i=1,2 .
$$

So $L_{1}$ and $L_{2}$ belong to a family of bundles parametrized by the space $\operatorname{Ext}\left(\mathcal{M} \otimes \mathcal{A}, \mathcal{A}^{\otimes(-1)}\right)$, which is connected, Q.E.D.

Remark. In the proof of Theorem 3 we used Proposition 5 in the particular case $Y=P^{1}$. In this case there is another proof of Proposition 5: use the well known theorem that all $G$-bundles on $P^{1}$ come from $H$ bundles, then compute the dimension of the automorphism group of each $G$-bundle and use the fact that all components of $\mathrm{Bun}_{G}$ have the same dimension $d=-\operatorname{dim} G$. 


\section{Acknowledgements}

The authors are grateful to A. Beauville and Y. Laszlo for a stimulating question and to A. A. Beilinson and V. A. Ginzburg for useful discussions.

\section{References}

1. A. Beauville and Y. Laszlo, Conformal blocks and generalized theta functions, Commun. Math. Physics 164 (1994), 385-419

2. A. Borel and T.A. Springer, Rationality properties of linear algebraic groups, Proc. Symp. Pure Math. A.M.S. 9 (1966), 26-32.

3. A. Borel and T.A. Springer, Rationality properties of linear algebraic groups II, Tôhoku Math. J. 20 (1968), 443-497.

4. M. Demazure and A. Grothendieck, Schémas en groupes, S.G.A. 3.

5. G. Faltings, A proof for the Verlinde formula, Journal of Algebraic Geometry 3 (1994), 347-374.

6. G. Harder, Halbeinfache Gruppenschemata über Dedekindringen, Invent. Math. 4 (1967), 165-191

7. Y. Laszlo and C. Sorger, The line bundles on the moduli of parabolic $G$-bundles over curves and their sections, to appear

8. C. S. Seshadri, Fibrés vectoriels sur les courbes algébriques, Astérisque 96 (1982)

9. R. Steinberg, Regular elements of semisimple algebraic groups, Publ. Math. IHES 25 (1965), 281-312

Physico-Technical Institute of Low Temperatures, Lenin Avenue 47, KHARKOV-164, 310164 Ukraine

Laboratoire Emile Picard, UFR-MiG, Université Paul Sabatier, 31062 Toulouse CEDEX, France

E-mail address: simpson@cict.fr 